\title{
Virtual Touch Quantification using Acoustic Radiation Force Impulse Imaging Technology versus Transient Elastography for the Noninvasive Assessment of Liver Fibrosis in Patients with Chronic Hepatitis B or C using Liver Biopsy as the Gold Standard
}

\author{
Victor Bâldea ${ }^{1}$, Ioan Sporea ${ }^{1}$, Anca Tudor ${ }^{2}$, Alina Popescu ${ }^{1}$, Felix Bende ${ }^{1}$, Roxana Sirli $^{1}$
}

\author{
1) Department of \\ Gastroenterology and \\ Hepatology, Victor Babeș \\ University of Medicine and \\ Pharmacy, Timişoara \\ 2) Department of Informatics \\ \& Medical Biostatistics, Victor \\ Babeș University of Medicine \\ and Pharmacy, Timișoara, \\ Romania
}

\begin{abstract}
Aims: Our aim was to assess the diagnostic performance of transient elastography (TE) and Virtual Touch Quantification (VTQ), a point Shear Wave Elastography (pSWE) technique, using Acoustic Radiation Force Impulse (ARFI) technology, for liver fibrosis assessment, as compared to percutaneous liver biopsy (LB), in patients with chronic hepatitis B or C.

Methods: We analyzed 157 patients ( 80 with chronic hepatitis B and 77 with chronic hepatitis C) with reliable liver stiffness (LS) measurements, in whom we compared TE and VTQ to the LB performed during the same session (evaluated according to the Metavir scoring system: F0-F4). LS was assessed by TE (FibroScan, EchoSens, Paris, France) and VTQ using the Siemens Acuson S2000TM ultrasound system (Siemens AG, Erlangen, Germany). We defined reliable LS measurements as the median value of 10 measurements with an IQR/M $<30 \%$ for both TE (obtained using the M probe) and VTQ. The areas under receiver operating characteristic curves (AUROCs) were used to assess the diagnostic performance of TE and VTQ. Correlation analysis determined the relationship between LSM values and liver histology.

Results: On LB 31 (19.7\%) patients had no fibrosis, 35 (22.3\%) had F1, 43 (27.4\%) had F2, 28 (17.8\%) had F3 and $20(12.7 \%)$ had cirrhosis. The mean size of the liver specimen in LB was $27 \mathrm{~mm}$. A strong, linear correlation (Spearman $\rho=0.826 ; \mathrm{p}<0.001)$ with $95 \%$ confidence interval for rho (0.769- 0.870), was found between the TE and VTQ measurements. By comparing the AUROC curves, TE and VTQ had similar predictive values for the presence of $F \geq 1$ Metavir: AUROC TE=0.876, AUROC VTQ=0.832, $\mathrm{p}=0.358$, for $\mathrm{F} \geq 2 \mathrm{Metavir}$ : AUROC $\mathrm{TE}=0.826$, AUROC VTQ=0.862, $\mathrm{p}=0.313$, for $\mathrm{F} \geq 3$ Metavir: $\mathrm{AUROC}$ TE=0.907, AUROC VTQ=0.880, $\mathrm{p}=0.434$ and for $\mathrm{F}=4$ Metavir: AUROC TE=0.981, AUROC VTQ=0.974, $\mathrm{p}=0.423$.

Conclusions: Both methods, TE and VTQ (pSWE) offer excellent diagnostic accuracy for liver fibrosis assessment in patients with chronic hepatitis $\mathrm{B}$ or $\mathrm{C}$ with similar performance.
\end{abstract}

Key words: transient elastography - virtual touch quantification - liver biopsy - HCV - HBV.

Abbreviations: ARFI: Acoustic Radiation Force Impulse; AUROC: area under the receiver operating characteristics; CLD: chronic liver diseases HBV: hepatitis B virus; HCV: hepatitis C virus; LB: percutaneous liver biopsy; LS: liver stiffness; pSWE: point shear wave elastography; ROI: region of interest; TE: transient elastography; VTQ: Virtual Touch Quantification.

\section{INTRODUCTION}

Chronic liver diseases (CLD) represent a significant public health problem. It is currently estimated that around 248 million people are chronically infected with hepatitis $B$ virus $(\mathrm{HBV})$ and 71 million people with hepatitis $\mathrm{C}$ virus (HCV) worldwide [1-3] and together, $\mathrm{HBV}$ and HCV account for $96 \%$ of viral hepatitis-related mortality [4]. Chronic hepatitis B and $\mathrm{C}$ commonly result in CLD with liver fibrosis representing the organ's final response to injury. Without effective treatment, this process can lead to cirrhosis, with the development of serious complications, such as portal hypertension, liver failure, and liver cancer. Therefore, it is invaluable to accurately diagnose and monitor the progression of hepatic fibrogenesis in each patient, for timely and effective prevention, treatment and progression of CLD.

Liver biopsy (LB) is still considered the gold standard in the assessment of hepatic fibrosis, allowing not only the ascertainment of fibrosis but also important parameters such 
as inflammation, necrosis, steatosis or presence of hepatic iron in the obtained specimens [5]. Despite its diagnostic utility, LB is limited because of its invasiveness and cost, the risk of complications, including death, its poor acceptance by patients, the lack of availability of expert practitioners, and intra/inter-observer variability [6-8]. The limitations and invasive nature of LB have spurred extensive research for the development of non-invasive tests to measure liver fibrosis in patients with CLD.

Ultrasound based elastography techniques have been developed for the non-invasive assessment of liver fibrosis. The first one was transient elastography (TE) (FibroScan, EchoSens, Paris, France), currently the most extensively used in clinical practice [9-12], followed by Acoustic Radiation Force Impulse elastography (ARFI) technology. Both methods have the advantage that the underlying principle is more obvious and appealing to practitioners, the results can be obtained immediately during an examination, have a relatively fast learning curve and are risk-free as compared to liver biopsy.

Many prospective studies have evaluated the performance of TE in staging liver fibrosis in patients with CLD, including chronic viral hepatitis B and $\mathrm{C}$, which have led to the acceptance of this method for fibrosis assessment by international guidelines $[13,14]$. Several meta-analyses have found that TE is highly accurate for the diagnosis of advanced fibrosis and cirrhosis $[10,15-17]$, suggesting that TE may be used instead of liver biopsy in most patients with chronic hepatitis C and B $[11,15,18,19]$. Transient elastography however, has several drawbacks, including its ability to assess only the right lobe of the liver and its inability to visualize the site of measurement and the need for specific equipment designed to perform only elastography. Other drawbacks of TE are represented by the fact that ascites and obesity are important limiting factors.

Virtual Touch Tissue Quantification (VTQ), a proprietary technology developed by Siemens Healthcare is the first available real-time measurement technique that utilizes ARFI imaging, which enables assessment of tissue stiffness through the measurement of shear wave speed. Virtual Touch Tissue Quantification is a point Shear Wave Elastography (pSWE) method, enabling the evaluation of liver stiffness in a specific area designated by the operator. The technology used for VTQ is incorporated in an ultrasound system allowing the examiner to visualize the morphology of the liver at the same time. Therefore, VTQ takes advantage of a conventional ultrasound image to choose the positioning of the region of interest (ROI) in both planes, enabling the examiner to adjust the depth of the measuring site, the ability to avoid liver masses or vessels. VTQ examination can be performed in patients with ascites. Several prospective studies and meta-analyses have found VTQ to correlate well with the stage of hepatic fibrosis [2029] and have demonstrated its performance for the diagnosis of advanced fibrosis and cirrhosis in patients with chronic hepatitis C and B [27-33].

Our study aimed to assess the diagnostic accuracy and agreement between TE and VTQ for liver fibrosis assessment in patients chronically infected with the HBV and HCV, using $\mathrm{LB}$ as the method of reference.

\section{METHODS}

\section{Study population}

This was a monocentric cross-sectional study conducted in the Department of Gastroenterology and Hepatology, Timișoara County University Hospital, Romania between January 2010 and November 2018 and included patients diagnosed with chronic HBV infection and chronic HCV infection in whom LB had been performed. The study was approved by the institutional review board and the Ethics Committee of Victor Babeș University of Medicine and Pharmacy Timișoara and was performed in accordance with the World Medical Association Declaration of Helsinki, revised in 2000, Edinburgh. Informed consent was obtained from all participating subjects.

Inclusion criteria were patients older than 18 years, diagnosed with chronic HBV infection or chronic HCV infection, willing to undergo TE and VTQ measurements. Chronic viral hepatitis was diagnosed when either HBV surface antigen and HBV-DNA, or HCV antibodies and HCV-RNA were present in the serum. No patient tested positive for hepatitis Delta antibodies.

Exclusion criteria were patients under 18 years old, undergoing antiviral treatment, with ascites, who were taking beta-blocker medication (e.g. propranolol, carvedilol), with signs of biliary obstruction or liver congestion secondary to heart failure, with focal liver lesions, with fatty infiltration and body mass index (BMI) $>30 \mathrm{~kg} / \mathrm{m}^{2}$, with heavy alcohol consumption (ethanol intake $>210 \mathrm{~g}$ per week for men and $>140$ g per week for women), known with primary biliary cholangitis, primary sclerosing cholangitis or autoimmune hepatitis, presenting elevation of aspartate aminotransferase (AST) and alanine aminotransferase (ALT) more than five times the upper limit of normal (ULN) values.

The demographic, clinical and biological information of all patients were obtained from their medical records. Data included patients' age, gender, BMI, complete blood counts, international normalized ratio (INR), total bilirubin concentrations, ALT and AST levels, serum albumin.

Transient Elastography and VTQ were performed on the same day, by different physicians, highly experienced and trained with each method and more than 2 years of experience in B-mode US. They were blinded to each other's results and to the LB results. After elastographic measurements, LB was performed, in less than a week interval. All biopsy specimens were analyzed by experienced pathologists (more than 15 years of experience), who were blinded to the patient's clinical results and elastographic measurements.

\section{Histological assessment}

Liver biopsy was performed ultrasound-assisted, using 1.4 and $1.6 \mathrm{~mm}$ Menghini type modified needles. Only LB fragments at least $2 \mathrm{~cm}$ in length were considered adequate for pathological assessment. The mean biopsy specimen length was $27 \mathrm{~mm}$. All biopsy specimens were examined by experienced pathologists (more than 15 years of experience) from the Pathology Department, Victor Babeș University of Medicine and Pharmacy, Timișoara. The classification score used for the 
analysis of biopsy specimens was the Metavir scoring system, using a five-point scale $[34,35]$ : stage F0 indicated no fibrosis, F1 portal fibrosis without septa, F2 portal fibrosis with a few septa, F3 numerous septa without cirrhosis and F4 cirrhosis.

\section{Transient Elastography}

Transient Elastography was performed in all patients using the FibroScan system (EchoSens, Paris, France), using the $\mathrm{M}$ probe (standard probe - transducer frequency 3.5 $\mathrm{MHz}$ ). The examinations were performed according to the EFSUMB and WFUMB guidelines [13, 14]: all patients fasted for at least 3 hours before the elastographic measurements were performed, with each patient in a supine position, right arm in maximum abduction, by intercostal approach, in the right liver lobe. In each patient, we aimed for 10 valid liver stiffness (LS) measurements. The median value of 10 valid LS measurements was calculated and the results were expressed in kilopascals $(\mathrm{kPa})$. Reliable measurements were defined as the median value of 10 valid LS measurements with an interquartile range interval/median ratio (IQR/M) $<30 \%$ $[13,14]$. Patients with invalid/unreliable measurements were excluded from the study.

\section{Virtual Touch Tissue Quantification}

Virtual Touch Tissue Quantification was performed in all patients using ARFI imaging technology implemented on the Siemens Acuson S2000 ultrasound system (Siemens AG, Erlangen, Germany), using an abdominal curved transducer (4C1). Liver stiffness measurements were performed according to the EFSUMB and WFUMB guidelines $[13,14]$ : each patient was positioned in a supine position, right arm in maximum abduction, by intercostal approach, in the right liver lobe (liver segments V, VII, VIII) and with the transducer at a 90-degree angle to the liver capsule, in an area free of large vessels. The ROI is a $5 \mathrm{~mm} \times 10 \mathrm{~mm}$ rectangle, which can be freely moved by the operator in a two-dimensional $\mathrm{B}$ mode to a maximum depth of $8 \mathrm{~cm}$. The ROI was placed at a depth of $2 \mathrm{~cm}$ from the liver capsule taking care to avoid any large vessels or areas with artifacts. The measurements were taken with minimal scanning pressure applied by the operator, while the patients were asked to stop breathing for few moments. Previously the patients were instructed to avoid deep inspiration or expiration, to minimize the breathing motion. The median value of 10 valid LS measurements was calculated and the results expressed in $\mathrm{m} / \mathrm{s}$ from VTQ were converted to the Young modulus and expressed in kiloPascals $(\mathrm{kPa})$ [36]. Reliable measurements were defined as the median value of 10 valid LS measurements with an interquartile range interval/median ratio (IQR/M) $<30 \%[13,14]$. Patients with invalid/unreliable measurements were excluded from the study.

\section{Statistical analysis}

The statistical analysis was made using SPSS v.17 and Med Calc software. Quantitative variables were expressed as mean (standard deviation), median and range, and compared using the non-parametric Kruskal-Wallis test statistics (for more than two groups) and the non-parametric Mann-Whitney U test (for two groups comparison). Nominal variables were expressed as frequencies and proportions and compared using the Chi-
Squared test. Chi-Squared analysis was used to determine whether technical success and reliable measurements of TE and VTQ were significantly different. To specify if the LS values measured by TE, respectively VTQ differ statistically significantly between the adjacent stages of fibrosis we applied the Wilcoxon Signed Ranks test. The performance of the non-invasive methods was estimated using ROC curves by identifying the optimal cut-off points of different degrees of liver fibrosis in terms of sensitivity and specificity. The area under the ROC curve (AUROC) indicates the accuracy of the studied methods. Correlations between TE and VTQ measurements were estimated using Spearman's correlation coefficient and its two-sided 95\% confidence interval (CI), as calculated with Fisher's z transformation. Agreements between "equivalent Metavir" fibrosis stages on VTQ and TE were assessed by inter-rater agreement (kappa); two-sided $95 \%$ CIs were also estimated. Kappa values $\geq 0.60$ and $\geq 0.80$ were considered indicative of good and very good agreement, respectively. Any value of $\mathrm{p}<0.05$ was considered statistically significant.

\section{RESULTS}

\section{Baseline characteristics}

Two-hundred-forty-five patients diagnosed with chronic $\mathrm{HCV}$ infection or chronic HBV infection were invited to participate in our study. Eighty-eight patients were excluded from the study (Fig. 1). Finally, a total of 157 subjects, with reliable LS measurements with TE and VTQ and LB were analyzed. Group characteristics are presented in Table I.

Table I. Baseline characteristics of patients with reliable liver stiffness measurements

\begin{tabular}{ll}
\hline Variables & Mean \pm SD \\
\hline Age (years) & $46.8 \pm 14.02$ \\
BMI $\left(\mathrm{kg} / \mathrm{m}^{2}\right)$ & $24.9 \pm 2.87$ \\
AST $(\mathrm{IU} / \mathrm{L})$ & $30 \pm 16.39$ \\
ALT $(\mathrm{IU} / \mathrm{L})$ & $35.3 \pm 20.64$ \\
Total bilirubin $(\mathrm{mg} / \mathrm{dL})$ & $1.0 \pm 0.11$ \\
Platelets x10 $\left(/ \mathrm{mm}^{3}\right)$ & $254.8 \pm 61.74$ \\
Albumin $(\mathrm{g} / \mathrm{dL})$ & $4.1 \pm 0.22$ \\
INR & $1.1 \pm 0.29$ \\
TE $(\mathrm{kPa})$ & $8.2 \pm 3.8$ \\
VTQ $(\mathrm{kPa})$ & $7.1 \pm 3.81$ \\
\hline
\end{tabular}

ALT: alanine aminotransferase; AST: aspartate aminotransferase; BMI: body mass index; INR: international normalized ratio; SD: standard deviation; TE: transient elastography; VTQ: Virtual Touch Quantification

In the final cohort of 157 patients, the distribution of fibrosis severity assessed according to the Metavir staging system was as follows: $19.7 \%$ (31 patients) had no fibrosis, $22.3 \%$ (35 patients) had $\mathrm{F} 1,27.4 \%$ (43 patients) had $\mathrm{F} 2,17.8 \%$ (28 patients) had F3 and $12.7 \%$ (20 patients) had cirrhosis.

Among the 169 patients who underwent LS measurements with TE and VTQ, TE failed to provide LS values in four patients, and VTQ in two patients. Failure of LSM was due to narrow intercostal spaces in five patients and to poor compliance in one patient. There was no significant difference 


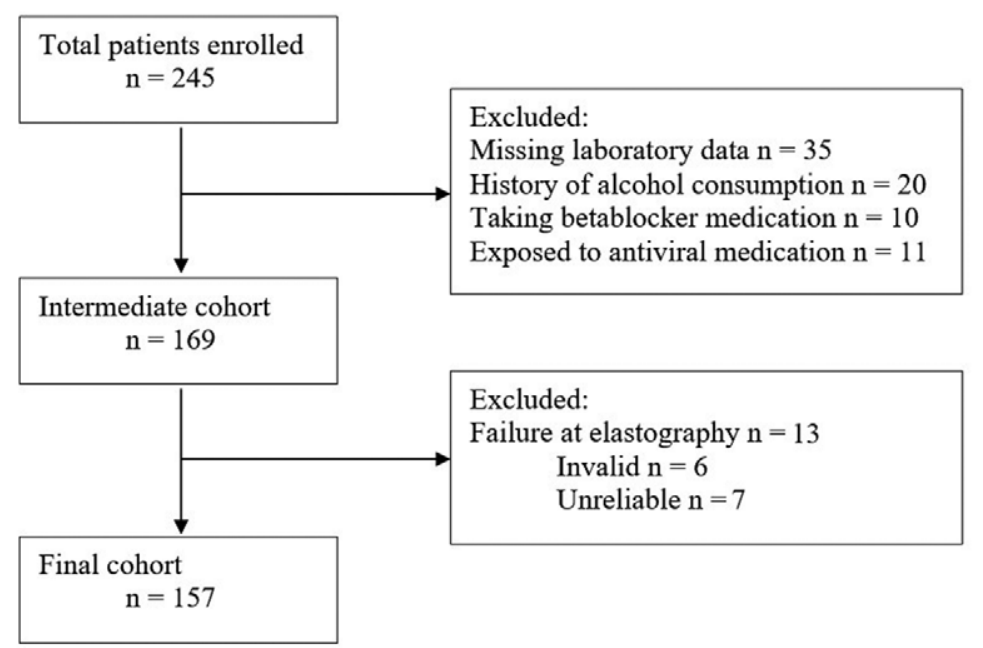

Fig. 1. Study flow diagram.

in the technical success rate of TE (97.6\% [165/169]) and VTQ $(98.8 \%$ [167/169]) $(\mathrm{p}=0.41)$. There were three unreliable LS measurement on TE and four on VTQ. The unreliable LSM with TE were due to narrow intercostal spaces, while with VTQ to narrow intercostal spaces in two patients, and poor compliance in two patients. All patients who had a technical failure on TE had reliable LSM on VTQ. One patient who had a technical failure on VTQ also had unreliable LSM on TE. There was no statistical difference between the reliable measurements of TE (98.2\% [166/169]) and VTQ (97.6\% [165/169]) ( $\mathrm{p=0.70).}$

The variability of different parameters according to fibrosis severity (Metavir) are presented in Table II. Higher age, AST values, ALT values, total bilirubin, INR, lower platelet count, and lower albumin were associated with increased fibrosis stage (Table II).

The median TE values were $6.7 \mathrm{kPa}$ for patients belonging to the $\mathrm{F} 1$ group, $7.3 \mathrm{kPa}$ for patients in the $\mathrm{F} 2$ group, $9.15 \mathrm{kPa}$ for patients in the $\mathrm{F} 3$ group and $14 \mathrm{kPa}$ for patients in the F4 group. The median VTQ values for patients belonging to the $\mathrm{F} 1$ group were $5.3 \mathrm{kPa}, 6.3 \mathrm{kPa}$ for patients in the F2 group, $7.2 \mathrm{kPa}$ in the $\mathrm{F} 3$ group and $13 \mathrm{kPa}$ for patients belonging to the F4 group. FibroScan and VTQ values significantly increase with increasing fibrosis stage according to the Metavir staging system, $\mathrm{p}<0.001$ (Figs. 2a and 2b). Using the Wilcoxon Signed Ranks Test we found that LS values measured by TE were significantly greater than those measured by VTQ, in the F0, F1, F2 and F3 Metavir stages ( $\mathrm{p}=0.013, \mathrm{p}<0.001, \mathrm{p}=0.008$ and $p=0.001)$, but not in the F4 Metavir stage $(p=0.232)$.

The diagnostic performance of TE and VTQ for predicting different stages of liver fibrosis, evaluated by ROC analysis are presented in Table III.

Pairwise comparisons of ROC curves between TE and VTQ showed no significant differences in their performance for staging fibrosis: $F \geq 1(p=0.358), F \geq 2(p=0.313), F \geq 3(p=0.434)$ and $\mathrm{F} 4$ fibrosis $(\mathrm{p}=0.423$ ) (Figs. 3a-d).

We found a significant, direct and strong correlation between TE and VTQ liver stiffness measurements (rho Spearman's coefficient $=0.826$, with $95 \%$ CI for rho: $0.769-0.870$, $\mathrm{p}<0.001$ ) (Fig. 4).

The association between fibrosis stage classification by the two diagnostic methods is statistically significant (Chi-squared Test, $\mathrm{p}<0.001)$. The inter-rater agreement (kappa) was 0.545 (moderate agreement), with $95 \% \mathrm{CI}$ for $\kappa(0.449,0.641)$, with an overall proportion of agreement of $51.59 \%$ (95\%CI: $43.49,65.71)$.

Table II. Variability of different parameters according to fibrosis severity (Metavir)

\begin{tabular}{|c|c|c|c|c|c|c|}
\hline \multirow[b]{2}{*}{ Variables } & \multicolumn{5}{|c|}{ Metavir grades } & \multirow[t]{2}{*}{$\mathrm{p}$} \\
\hline & F0 & $\mathrm{F} 1$ & $\mathrm{~F} 2$ & F3 & $\mathrm{F} 4$ & \\
\hline Age (years) mean \pm SD & $41.5 \pm 12.24$ & $41.1 \pm 2.15$ & $48.8 \pm 14.62$ & $49.1 \pm 13.53$ & $57.3 \pm 11.84$ & $<0.001$ \\
\hline BMI $\left(\mathrm{kg} / \mathrm{m}^{2}\right)$ mean $\pm \mathrm{SD}$ & $24.5 \pm 3.21$ & $24.6 \pm 2.86$ & $25.3 \pm 2.77$ & $25.0 \pm 2.78$ & $25.1 \pm 2.81$ & 0.866 \\
\hline $\mathrm{AST}(\mathrm{IU} / \mathrm{L})$ mean $\pm \mathrm{SD}$ & $22.3 \pm 7.93$ & $25.1 \pm 10.15$ & $34.3 \pm 17.21$ & $38.2 \pm 23.14$ & $29.5 \pm 14.89$ & $<0.001$ \\
\hline $\operatorname{ALT}(\mathrm{IU} / \mathrm{L})$ mean $\pm \mathrm{SD}$ & $29.0 \pm 14.83$ & $31.3 \pm 17.69$ & $40.7 \pm 22.74$ & $44.2 \pm 25.74$ & $28.2 \pm 13.17$ & 0.013 \\
\hline $\begin{array}{l}\text { Total bilirubin }(\mathrm{mg} / \mathrm{dL}) \\
\text { mean } \pm \mathrm{SD}\end{array}$ & $1.0 \pm 0.07$ & $1.0 \pm 0.05$ & $1.0 \pm 0.05$ & $1.0 \pm 0.11$ & $1.2 \pm 0.16$ & $<0.001$ \\
\hline Platelets $\times 10^{3}\left(/ \mathrm{mm}^{3}\right)$ mean \pm SD & $265.6 \pm 49.5$ & $261.5 \pm 59.53$ & $269.0 \pm 65.58$ & $256.0 \pm 51.89$ & $193.8 \pm 55.68$ & $<0.001$ \\
\hline Albumin $(\mathrm{g} / \mathrm{dL})$ mean $\pm \mathrm{SD}$ & $4.1 \pm 0.22$ & $4.1 \pm 0.19$ & $4.1 \pm 0.24$ & $4.1 \pm 0.2$ & $4.0 \pm 0.25$ & 0.049 \\
\hline INR mean \pm SD & $1.1 \pm 0.28$ & $1.1 \pm 0.3$ & $1.0 \pm 0.25$ & $1.1 \pm 0.3$ & $1.2 \pm 0.34$ & 0.042 \\
\hline $\mathrm{TE}(\mathrm{kPa})$ mean $\pm \mathrm{SD}$ & $5.3 \pm 1.19$ & $6.8 \pm 1.38$ & $7.2 \pm 1.52$ & $9.3 \pm 2.13$ & $15.5 \pm 4.89$ & $<0.001$ \\
\hline VTQ $(\mathrm{kPa})$ mean $\pm \mathrm{SD}$ & $4.6 \pm 1.73$ & $5.4 \pm 1.66$ & $6.5 \pm 1.44$ & $7.7 \pm 2.01$ & $14.5 \pm 4.93$ & $<0.001$ \\
\hline
\end{tabular}

For abbreviations see Table I 

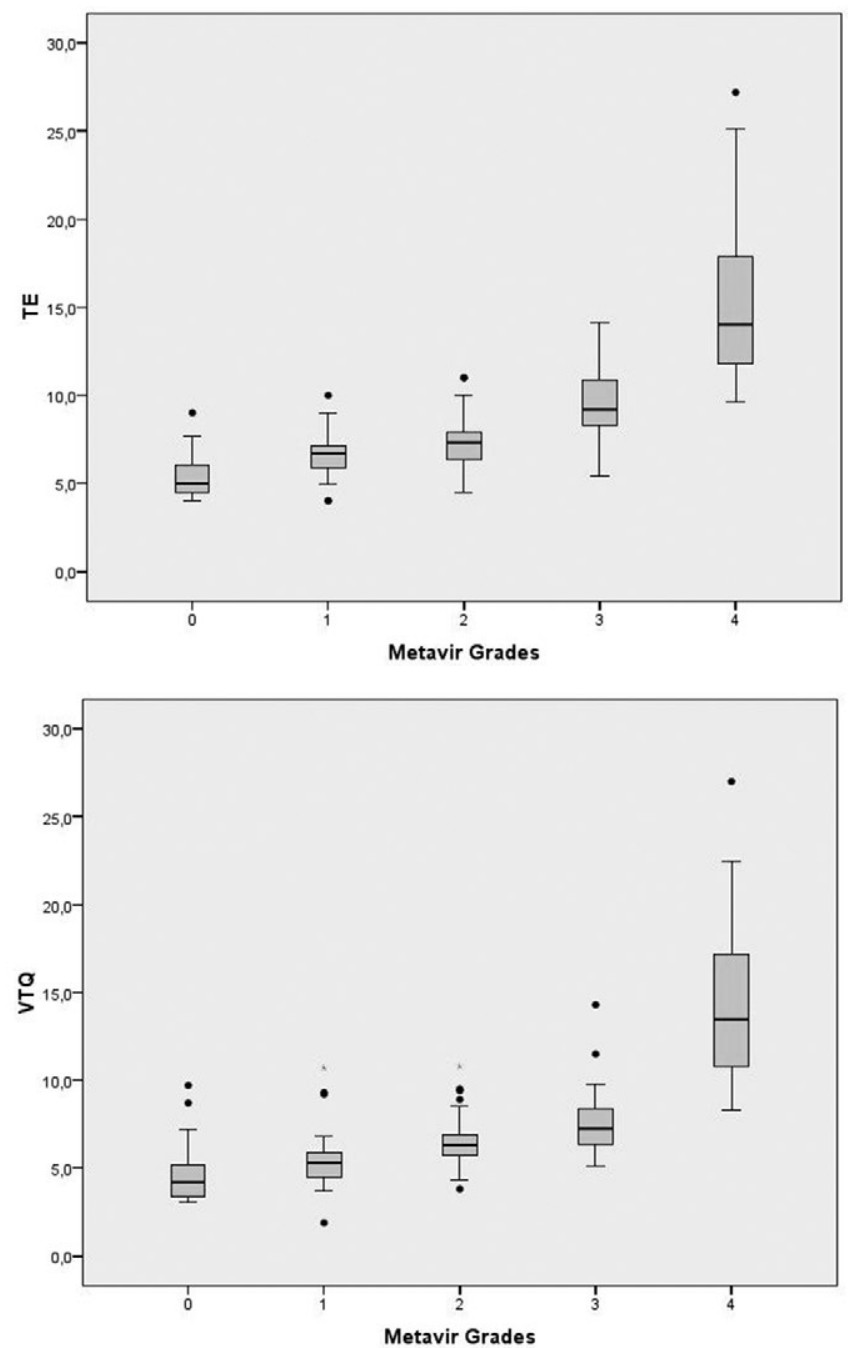

Fig. 2. Median liver stiffness values according to fibrosis severity evaluated by TE (Fig.2a) and by VTQ (Fig. 2b).

Using the TE cut-off value of $7.05 \mathrm{kPa}$ for predicting significant fibrosis (Metavir $\mathrm{F} \geq 2$ ), $8.1 \mathrm{kPa}$ for the diagnosis of severe fibrosis (Metavir $\mathrm{F} \geq 3$ ) and $9.55 \mathrm{kPa}$ for the diagnosis of cirrhosis (Metavir $\mathrm{F}=4$ ), we evaluated the concordance rate of TE vs histological diagnosis of liver fibrosis. A cumulative concordance rate of $56.1 \%$ was found $(\kappa=0.608$; $95 \%$ CI: 0.523 0.693) (Table IV). We performed the same analysis using the VTQ cut-off value of $5.55 \mathrm{kPa}$ for predicting significant fibrosis (Metavir $\mathrm{F} \geq 2$ ), $6.9 \mathrm{kPa}$ for the diagnosis of severe fibrosis (Metavir $\mathrm{F} \geq 3$ ) and $8.25 \mathrm{kPa}$ for the diagnosis of cirrhosis (Metavir $\mathrm{F}=4$ ). The cumulative rate of concordance was of 54.1\% ( $\kappa=0.587$; 95\%CI: 0.498-0.675) (Table IV).

Of the 157 patients, 69 (43.9\%) showed discordance between the TE and Metavir score, while 72 (45.8\%) showed discordance between the VTQ and Metavir score. Several factors possibly associated with these discordances were analyzed, including age, gender, BMI, ALT, AST, biopsy specimen length. Only biopsy specimen length was associated with the discordance between the TE and Metavir score $(\mathrm{p}=0.026)$, respectively VTQ and Metavir score $(\mathrm{p}=0.034)$.

\section{DISCUSSION}

The correct evaluation of liver fibrosis in patients with chronic HBV or HCV infection is of paramount importance for patient management, especially with the introduction of potent direct-acting agents (DAA), which can cure chronic $\mathrm{HCV}$ infection with a very high success rate following an 8-24 week course of treatment and the existence of potent nucleoside/nucleotide analogs that can control almost all chronic HBV infected patients. The presence of significant fibrosis is an indication for urgent treatment, whereas the presence of severe fibrosis and cirrhosis is an indication for initiating a surveillance program.

Several factors can influence the results of non-invasive methods for liver fibrosis assessment. A possible explanation for our results, the increased LS values measured by TE when compared to VTQ, could lie in the underlying technology used to generate shear waves, in the case of TE being a low-frequency mechanical vibration, while VTQ uses high-frequency US

Table III. TE and VTQ Cut-off values and performance for the diagnosis of fibrosis stage according to the Metavir staging system

\begin{tabular}{|c|c|c|c|c|c|c|}
\hline & $\begin{array}{l}\text { Cutoff } \\
(\mathrm{kPa})\end{array}$ & $\begin{array}{c}\text { AUC } \\
(95 \% \mathrm{CI})\end{array}$ & $\begin{array}{c}\text { Sensitivity }(\%) \\
(95 \% \mathrm{CI})\end{array}$ & $\begin{array}{c}\text { Specificity }(\%) \\
(95 \% \mathrm{CI})\end{array}$ & $\begin{array}{l}\text { PPV (\%) } \\
(95 \% \mathrm{CI})\end{array}$ & $\begin{array}{l}\text { NPV (\%) } \\
(95 \% \mathrm{CI})\end{array}$ \\
\hline \multicolumn{7}{|c|}{$\mathrm{F} \geq 1$ (prevalence $80.3 \%)$} \\
\hline $\mathrm{TE}$ & 6.05 & $0.876(0.814,0.923)$ & $82.5(74.8,88.7)$ & $83.9(66.3,94.5)$ & $95.4(88.6,99.8)$ & $54.2(47.4,61.0)$ \\
\hline VTQ & 5.05 & $0.832(0.765,0.887)$ & $84.1(76.6,90.0)$ & $74.2(55.4,88.1)$ & $93(86.2,99.8)$ & $53.5(46.7,63)$ \\
\hline \multicolumn{7}{|c|}{$\mathrm{F} \geq 2$ (prevalence $57.9 \%)$} \\
\hline $\mathrm{TE}$ & 7.05 & $0.826(0.757,0.882)$ & $72.5(62.2,81.4)$ & $81.8(70.4,90.2)$ & $84.6(68.2,81.8)$ & $68.4(61.6,75.2)$ \\
\hline VTQ & 5.55 & $0.862(0.798,0.912)$ & $87.9(79.4,93.8)$ & $72.7(60.4,83.0)$ & $81.6(62.3,75.9)$ & $81.4(74.6,88.2)$ \\
\hline \multicolumn{7}{|c|}{$\mathrm{F} \geq 3$ (prevalence $30.6 \%)$} \\
\hline $\mathrm{TE}$ & 8.1 & $0.907(0.851,0.948)$ & $87.5(74.8,95.3)$ & $87.2(79.4,92.8)$ & $75(68.2,81.8)$ & $94.1(87.3,99.9)$ \\
\hline VTQ & 6.9 & $0.88(0.819,0.927)$ & $79.2(65.0,89.5)$ & $84.4(76.2,90.6)$ & $69.1(62.3,75.9)$ & $92(83.4,97.0)$ \\
\hline \multicolumn{7}{|c|}{$\mathrm{F}=4($ prevalence $12.7 \%)$} \\
\hline $\mathrm{TE}$ & 9.55 & $0.981(0.945,0.996)$ & $100(83.2,100.0)$ & $88.3(81.7,93.2)$ & $55.5(48.7,62.3)$ & $97.7(92.9,100)$ \\
\hline VTQ & 8.25 & $0.974(0.935,0.993)$ & $100(83.2,100.0)$ & $85.4(78.4,90.8)$ & $50(43.2,56.8)$ & $98.2(92.4,99.9)$ \\
\hline
\end{tabular}

CI: confidence intervals; PPV: positive predictive value, NPV: negative predictive value; TE: transient elastography; VTQ: Virtual Touch Quantification 

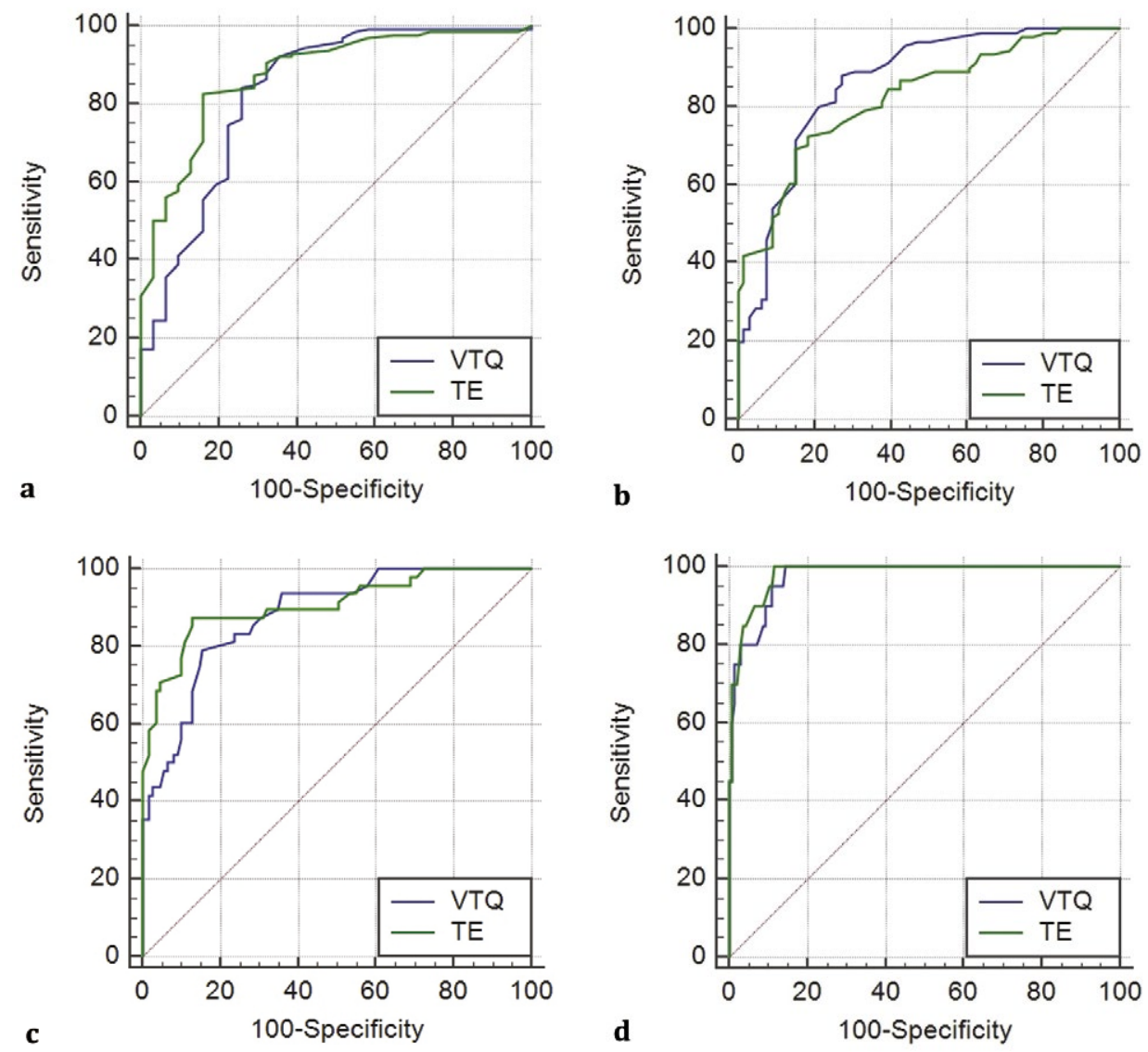

Fig. 3. Comparative performance (evaluated by receiver operating characteristic curves analysis) of TE and VTQ to predict different stages of fibrosis (Fig.3a: F $\geq 1$; Fig.3b: F $\geq 2$; Fig.3c: F $\geq 3$; Fig.3d: F=4).

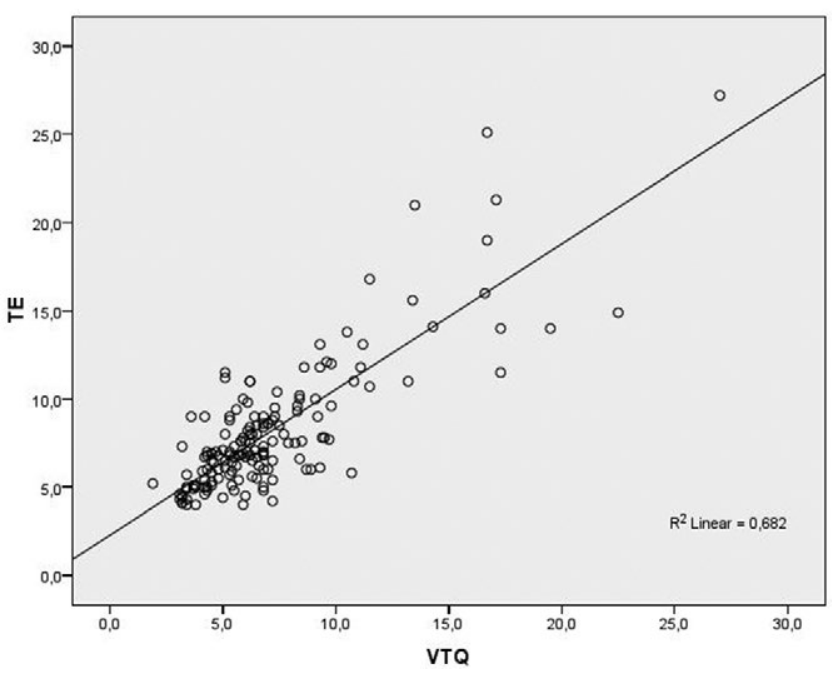

Fig. 4. Correlation between TE and VTQ liver stiffness measurements (rho Spearman's coefficient $=0.826$, with $95 \% \mathrm{CI}$ for rho: $0.769-0.870$, $\mathrm{p}<0.001$.

„push pulse”. Our results are in accordance with other studies, where mean LS values obtained by pSWE systems were lower than those obtained by TE in the lower spectrum of fibrosis, whereas in cases of liver cirrhosis, although the variability of LS measurements obtained was higher, the concordance rate between them followed the same direction [37].

Regarding TE, the $M$ transducer was used for all TE examinations, to avoid potential bias, because when both the $\mathrm{M}$ and XL probes are used, different results can occur [38-40], although some studies have suggested no significant differences between results obtained with the M or XL probe if the appropriate probe was used according to the automatic probe selection [41]. We also accounted for other factors such as platelet count, level of aminotransferases, albumin, BMI, presence of diabetes mellitus type 2, severe steatosis, betablocker medication, that can influence LS measurements [12, 42-44]. Regarding VTQ, a skin-liver distance $>2.5 \mathrm{~cm}$ and a high BMI were taken as confounding factors as they increase the discrepancy when compared to liver biopsy $[45,46]$.

Our study has shown that these two methods yielded similar results. VTQ and TE LS measurements showed a very good correlation when compared $(\mathrm{p}<0.001$, rho Spearman's coefficient $=0.826$, with $95 \% \mathrm{CI}$ for rho: $0.769-0.870$ ). We also found a moderate agreement between the two methods, kappa $=0.545,95 \%$ CI: $0.449-0.641$ and a concordance rate of $51.59 \%$, which is similar to a previously reported study performed by Rizzo L et al. [47] who found a concordance rate of $54.7 \%$.

In this study $\mathrm{TE}$ was consistently accurate in classifying stages of fibrosis as $\mathrm{F} \geq 1, \mathrm{~F} \geq 2, \mathrm{~F} \geq 3$ or $\mathrm{F}=4$, with AUROCs of $0.87,0.82,0.90,0.97$ which was consistent with the findings of the literature (Table V).

In our study, VTQ had AUROCs of 0.83 for $F \geq 1,0.86$ for $\mathrm{F} \geq 2,0.88$ for $\mathrm{F} \geq 3$, and 0.97 for $\mathrm{F}=4$, results consistent with the findings of the meta-analyses performed by Friedrich-Rust et al. [51] and Nierhoff et al. [33] (Table VI). 
Table IV. Analysis of concordance of TE and VTQ versus Metavir stage

\begin{tabular}{|c|c|c|c|c|c|c|c|}
\hline & F0 & $\mathrm{F} 1$ & F2 & F3 & $\mathrm{F} 4$ & Total & $\begin{array}{c}\text { Concordance } \\
\text { rate }(\%)\end{array}$ \\
\hline \multicolumn{8}{|l|}{ F0 } \\
\hline $\mathrm{TE}<6.05 \mathrm{kPa}$ & 26 & 10 & 10 & 2 & 0 & 48 & 54.1 \\
\hline $\mathrm{VTQ}<5.05 \mathrm{kPa}$ & 23 & 15 & 5 & 0 & 0 & 43 & 53.4 \\
\hline \multicolumn{8}{|l|}{ F1 } \\
\hline $6.05 \mathrm{kPa} \leq \mathrm{TE}<7.05 \mathrm{kPa}$ & 2 & 16 & 10 & 3 & 0 & 31 & 51.6 \\
\hline $5.05 \mathrm{kPa} \leq \mathrm{VTQ}<5.55 \mathrm{kPa}$ & 1 & 9 & 3 & 3 & 0 & 16 & 56.2 \\
\hline \multicolumn{8}{|l|}{$\mathrm{F} 2$} \\
\hline $7.05 \mathrm{kPa} \leq \mathrm{TE}<8.1 \mathrm{kPa}$ & 2 & 3 & 16 & 1 & 0 & 22 & 72.7 \\
\hline $5.55 \mathrm{kPa} \leq \mathrm{VTQ}<6.9 \mathrm{kPa}$ & 4 & 8 & 24 & 7 & 0 & 43 & 55.8 \\
\hline \multicolumn{8}{|l|}{ F3 } \\
\hline $8.1 \mathrm{kPa} \leq \mathrm{TE}<9.55 \mathrm{kPa}$ & 1 & 5 & 4 & 10 & 0 & 20 & 50.0 \\
\hline $6.9 \mathrm{kPa} \leq \mathrm{VTQ}<8.25 \mathrm{kPa}$ & 1 & 0 & 5 & 9 & 0 & 15 & 60.0 \\
\hline \multicolumn{8}{|l|}{$\mathrm{F} 4$} \\
\hline $\mathrm{TE} \geq 9.55 \mathrm{kPa}$ & 0 & 1 & 3 & 12 & 20 & 36 & 55.5 \\
\hline $\mathrm{VTQ} \geq 8.25 \mathrm{kPa}$ & 2 & 3 & 6 & 9 & 20 & 40 & 50.0 \\
\hline \multicolumn{8}{|l|}{ Cumulative concordance } \\
\hline TE & & \multicolumn{4}{|c|}{$\mathrm{k}=0.608$; 95\%CI: $0.523-0.693$} & 88 & 56.1 \\
\hline VTQ & & \multicolumn{4}{|c|}{$\mathrm{k}=0.587 ;$; $95 \%$ CI: $0.498-0.675$} & 85 & 54.1 \\
\hline
\end{tabular}

Table V. Performance of TE for staging liver fibrosis in previous studies

\begin{tabular}{lcccc}
\hline Study year, reference & $\begin{array}{c}\text { Fibrosis } \\
\text { stage }\end{array}$ & AUC & $\begin{array}{c}\text { Sensitivity } \\
(\%)\end{array}$ & $\begin{array}{c}\text { Specificity } \\
(\%)\end{array}$ \\
\hline Ziol, 2004 [48] & $\mathrm{F} \geq 2$ & 0.79 & 56 & 91 \\
& $\mathrm{~F} \geq 3$ & 0.96 & 86 & 85 \\
& $\mathrm{~F}=4$ & 0.97 & 86 & 96 \\
Castera, 2005 [49] & $\mathrm{F} \geq 2$ & 0.83 & 67 & 89 \\
& $\mathrm{~F} \geq 3$ & 0.90 & 73 & 91 \\
& $\mathrm{~F}=4$ & 0.95 & 87 & 91 \\
Ragazzo, 2017 [50] & $\mathrm{F} \geq 2$ & 0.83 & 71 & 92 \\
& $\mathrm{~F} \geq 3$ & 0.85 & 80 & 79 \\
& $\mathrm{~F}=4$ & 0.99 & 100 & 99 \\
\hline
\end{tabular}

Table VI. Performance of VTQ for staging liver fibrosis in previous studies

\begin{tabular}{lcc}
\hline Study & Fibrosis Stage & AUC \\
\hline Friedrich-Rust, 2011 [51] & $\mathrm{F} \geq 2$ & 0.87 \\
& $\mathrm{~F} \geq 3$ & 0.91 \\
& $\mathrm{~F}=4$ & 0.93 \\
Nierhoff, 2013 [33] & $\mathrm{F} \geq 2$ & 0.84 \\
& $\mathrm{~F} \geq 3$ & 0.89 \\
& $\mathrm{~F}=4$ & 0.91 \\
\hline
\end{tabular}

These results indicate that both methods are good tools for significant fibrosis and excellent for advanced fibrosis and cirrhosis. These findings are also consistent with the results from the meta-analysis performed by Bota et al. [27], who found TE and VTQ to be equally accurate in diagnosing significant fibrosis and cirrhosis.

We observed a moderate agreement between the two techniques for predicting $\mathrm{F} \geq 2$. In our study, the AUROCs of TE and VTQ were higher for predicting $\mathrm{F}=4$ than $\mathrm{F} \geq 2$ (AUROC
TE of 0.87 for $\mathrm{F} \geq 2$, respectively 0.97 for $\mathrm{F}=4$ and AUROC VTQ of 0.86 for $F \geq 2$, respectively 0.97 for $F=4$ ). Earlier studies also showed that TE or VTQ was more accurate for predicting extreme stages of liver fibrosis $(\mathrm{F} \geq 1$ or $\mathrm{F}=4)$ than $\mathrm{F} \geq 2[10,16$, $18,20,23,28]$.

We performed pairwise comparisons of ROC curves between TE and VTQ and were able to demonstrate that there are no significant differences in their performance for staging $\mathrm{F} \geq 1$ fibrosis $(\mathrm{p}=0.358), \mathrm{F} \geq 2$ fibrosis $(\mathrm{p}=0.313), \mathrm{F} \geq 3$ fibrosis $(\mathrm{p}=0.434)$ and $\mathrm{F}=4$ fibrosis $(\mathrm{p}=0.423)$. Our study revealed that both methods have similar performance in diagnosing all stages of liver fibrosis, results which are consistent with other studies that found equivalency between TE and VTQ for diagnosing all degrees of liver fibrosis [27, 52, 53], while other studies have found TE to be better for the diagnosis of significant fibrosis [23], and VTQ for both significant and severe classes of liver fibrosis $[47,50]$.

The optimal TE and VTQ cut-offs values for staging liver fibrosis from our study are presented in Table III and IV. We are aware that the TE cut-offs reported in our study are lower than the cut-off values reported in previous meta-analyses for diagnosing different fibrosis stages: for $\mathrm{F} \geq 2: 7.6 \mathrm{kPa}$ (range 5.1-10.1), for $\mathrm{F} \geq 3$ : $10.9 \mathrm{kPa}$ (range 8.0-15.4) and for $\mathrm{F}=4$ : $15.3 \mathrm{kPa}$ (range 11.9-26.5) in chronic hepatitis $\mathrm{C}$, whereas in chronic hepatitis $\mathrm{B}$ for $\mathrm{F} \geq 2: 7.0 \mathrm{kPa}$ (range 6.9-7.2), for $\mathrm{F} \geq 3$ : $8.2 \mathrm{kPa}$ (range 7.3-9.0) and for $\mathrm{F}=4: 11.3 \mathrm{kPa}$ (range 9.0-13.4), respectively $[10,18]$. The lower cut-off values reported could be explained by the small number of patients in our study and the low prevalence of liver cirrhosis. Another explanation could be our inclusion criteria (patients with ALT and AST values $<100 \mathrm{IU} / \mathrm{L}$, non-obese), our exclusion criteria (patients with known type 2 diabetes mellitus, patients exposed to antiviral treatment, beta-blocker treatment) and performance of TE measurements using only the $\mathrm{M}$ probe, all being confounding 
factors that can influence elastographic measurements, hence the heterogenicity of reported cut-off values from previous meta-analyses $[9,10,12]$.

In our study, we reported cut-off values expressed in $\mathrm{kPa}$ and to our knowledge, only one study reported their results in the same way [54]. In this study, Ryu H et al. [54] reported the mean VTQ values to be lower than TE values (10.5 kPa versus. $15.1 \mathrm{kPa}, \mathrm{p}<0.001)$, results which are consistent to our study.

Finally, we must take into consideration that VTQ is an ultrasound-based elastography method, allowing direct visualization of liver parenchyma, enabling simultaneous morphological and Doppler analysis of the liver, screening for focal liver lesions and evaluation of liver fibrosis. Moreover, the presence of the $\mathrm{B}$ mode allows the examiner to position the ROI in an area of preference, devoid of liver vessels or masses, with no measurement limitations due to overweight or the presence of ascites.

A limitation of our study is the relatively small number of patients, with a low prevalence of liver cirrhosis, which could have had an impact on the results. A further methodological limitation could reside in the accuracy of liver biopsy examination for assessing fibrosis, which is still defined as a 'gold standard", although we included biopsy specimens $>2$ $\mathrm{cm}$ in length to minimize the limitations previously discussed.

In our study, biopsy specimen length was associated with the discordance between TE and Metavir score $(\mathrm{p}=0.026)$, respectively the VTQ and Metavir score $(\mathrm{p}=0.034)$, which could be explained by the fact that fibrosis is spread heterogeneously within the liver during the progression of chronic liver disease, and can lead to sampling error, since liver biopsy cannot always be adequate for assessing liver fibrosis [6-8].

The strength in our study lies in the fact that we accounted for several factors that can impact elastographic measurements, included non-obese, non-drinker patients, who were enrolled in a tertiary referral center for liver diseases.

\section{CONCLUSION}

Our study offers sufficient evidence to consider VTQ (a pSWE method) a valuable alternative to TE for the noninvasive assessment of liver fibrosis in patients with HCV or HBV chronic infection.

Conflicts of interest: None to declare.

Authors' contribution: V.B. and I.S. conceived and designed the study. V.B. and F.B. collected the data. A.T. performed: statistical analysis. V.B, I.S., A.P. and R.Ș. analyzed the data and drafted the manuscript. All authors critically revised the manuscript, approved the final version and agreed to be accountable for all aspects of the work.

Acknowledgments: We thank Assoc. Prof. Mărioara Cornianu and Diana Hermann for the biopsy specimens histological analysis.

\section{REFERENCES}

1. Schweitzer A, Horn J, Mikolajczyk RT, Krause G, Ott JJ. Estimations of worldwide prevalence of chronic hepatitis B virus infection: a systematic review of data published between 1965 and 2013. Lancet 2015;386:15461555. doi:10.1016/s0140-6736(15)61412-x

2. Polaris Observatory HCV Collaborators. Global prevalence and genotype distribution of hepatitis $\mathrm{C}$ virus infection in 2015: a modelling study. Lancet Gastroenterol Hepatol 2017;2:161-176. doi:10.1016/s24681253(16)30181-9

3. Mason LM, Duffell E, Veldhuijzen IK, Petriti U, Bunge EM, Tavoschi L. Hepatitis B and C prevalence and incidence in key population groups with multiple risk factors in the EU/EEA: a systematic review. Euro Surveill 2019;24(30). doi:10.2807/1560-7917. es.2019.24.30.1800614

4. Stanaway JD, Flaxman AD, Naghavi M, et al. The global burden of viral hepatitis from 1990 to 2013: findings from the Global Burden of Disease Study 2013. Lancet 2016;388:1081-1088. doi:10.1016/s01406736(16)30579-7

5. Sebastiani G, Alberti A. Non invasive fibrosis biomarkers reduce but not substitute the need for liver biopsy. World J Gastroenterol 2006;12:36823694. doi:10.3748/wjg.v12.i23.3682

6. Cadranel JF, Rufat P, Degos F. Practices of Liver Biopsy in France: Results of a Prospective Nationwide Survey. Hepatology 2000;32:477481. doi:10.1053/jhep.2000.16602

7. Regev A, Berho M, Jeffers LJ, et al. Sampling error and intraobserver variation in liver biopsy in patients with chronic HCV infection. Am J Gastroenterol 2002;97:2614-2618.

8. Bedossa P, Dargere D, Paradis V. Sampling variability of liver fibrosis in chronic hepatitis C. Hepatology 2003;38:1449-1457. doi:10.1016/j. hep.2003.09.022

9. Talwalkar JA, Kurtz DM, Schoenleber SJ, West CP, Montori VM Ultrasound-based transient elastography for the detection of hepatic fibrosis: systematic review and meta-analysis. Clin Gastroenterol Hepatol 2007;5:1214-1220. doi:10.1016/j.cgh.2007.07.020

10. Friedrich-Rust M, Ong ME, Martens S et al. Performance of transient elastography for the staging of liver fibrosis: a metaanalysis. Gastroenterology 2008;134:960-974.e8. doi:10.1053/j. gastro.2008.01.034

11. Jung KS, Kim SU. Clinical applications of transient elastography. Clin Mol Hepatol 2012;18:163-173. doi:10.3350/cmh.2012.18.2.163

12. Castéra L, Foucher J, Bernard PH, et al. Pitfalls of liver stiffness measurement: A 5-year prospective study of 13,369 examinations. Hepatology 2010;51:828-835. doi:10.1002/hep.23425

13. Dietrich CF, Bamber J, Berzigotti A, et al. EFSUMB Guidelines and Recommendations on the Clinical Use of Liver Ultrasound Elastography, Update 2017 (Long Version). Ultraschall Med 2017;38:e16-e47. doi:10.1055/s-0043-103952

14. Ferraioli G, Filice C, Castera L, et al. WFUMB Guidelines and Recommendations for Clinical Use of Ultrasound Elastography: Part 3: Liver. Ultrasound Med Biol 2015;41:1161-1179. doi:10.1016/j. ultrasmedbio.2015.03.007

15. Li Y, Huang YS, Wang ZZ, et al. Systematic review with meta-analysis: the diagnostic accuracy of transient elastography for the staging of liver fibrosis in patients with chronic hepatitis B. Aliment Pharmacol Ther 2015;43:458-469. doi:10.1111/apt.13488

16. Tsochatzis EA, Gurusamy KS, Ntaoula S, Cholongitas E, Davidson BR, Burroughs AK. Elastography for the diagnosis of severity of fibrosis in chronic liver disease: A meta-analysis of diagnostic accuracy. J Hepatol 2011;54:650-659. doi:10.1016/j.jhep.2010.07.033

17. Xiao H, Shi M, Xie Y, Chi X. Comparison of diagnostic accuracy of magnetic resonance elastography and Fibroscan for detecting liver fibrosis in chronic hepatitis B patients: A systematic review and 
meta-analysis. PLoS One 2017;12:e0186660. doi:10.1371/journal. pone. 0186660

18. Castera L, Forns X, Alberti A. Non-invasive evaluation of liver fibrosis using transient elastography. J Hepatol 2008;48:835-847. doi:10.1016/j. jhep.2008.02.008

19. Huang R, Jiang N, Yang R, et al. Fibroscan improves the diagnosis sensitivity of liver fibrosis in patients with chronic hepatitis B. Exp Ther Med 2016;11:1673-1677. doi:10.3892/etm.2016.3135

20. Friedrich-Rust M, Wunder K, Kriener S, et al. Liver Fibrosis in Viral Hepatitis: Noninvasive Assessment with Acoustic Radiation Force Impulse Imaging versus Transient Elastography. Radiology 2009;252:595-604. doi:10.1148/radiol.2523081928

21. Lupșor M, Badea R, Stefanescu H, et al. Performance of a new elastographic method (ARFI technology) compared to unidimensional transient elastography in the non invasive assessment of chronic hepatitis C. Preliminary results. J Gastrointestin Liver Dis 2009;18:303310.

22. Fierbințeanu-Braticevici C, Andronescu D, Usvat R, Cretoiu D, Băicus C, Marinoschi G. Acoustic radiation force imaging sonoelastography for noninvasive staging of liver fibrosis. World J Gastroenterol 2009;15:5525-5532. doi:10.3748/wjg.15.5525

23. Sporea I, Șirli RL, Deleanu A, et al. Acoustic radiation force impulse elastography as compared to transient elastography and liver biopsy in patients with chronic hepatopathies. Ultraschall Med 2010;32(Suppl 1):S46-S52. doi:10.1055/s-0029-1245360

24. Takahashi H, Ono N, Eguchi Y, et al. Evaluation of acoustic radiation force impulse elastography for fibrosis staging of chronic liver dis ease: a pilot study. Liver Int 2010;30:538-545. doi:10.1111/j.14783231.2009.02130.x

25. Rifai K, Cornberg J, Mederacke I, et al. Clinical feasibility of liver elastography by acoustic radiation force impulse imaging (ARFI). Dig Liver Dis 2011;43:491-497. doi:10.1016/j.dld.2011.02.011

26. Cassinotto C, Lapuyade B, Aït-Ali A, et al. Liver fibrosis: noninvasive assessment with acoustic radiation force impulse elastographycomparison with FibroScan M and XL probes and FibroTest in patients with chronic liver disease. Radiology 2013;269:283-292. doi:10.1148/ radiol.13122208

27. Bota S, Herkner H, Sporea I et al. Meta-analysis: ARFI elastography versus transient elastography for the evaluation of liver fibrosis. Liver Int 2013;33:1138-1147. doi:10.1111/liv.12240

28. Hu X, Qiu L, Liu D, Qian L. Acoustic Radiation Force Impulse (ARFI) Elastography for noninvasive evaluation of hepatic fibrosis in chronic hepatitis B and C patients: a systematic review and meta-analysis. Med Ultrason 2017;19(1):23-31. doi:10.11152/mu-942

29. Kobayashi K, Nakao H, Nishiyama T, et al. Diagnostic Accuracy of Real-Time Tissue Elastography for the Staging of Liver Fibrosis: A Meta-Analysis. Eur Radiol 2014;25:230-238. doi:10.1007/s00330-0143364-x

30. Park MS, Kim SW, Yoon KT, et al. Factors Influencing the Diagnostic Accuracy of Acoustic Radiation Force Impulse Elastography in Patients with Chronic Hepatitis B. Gut Liver 2016;10:275-282. doi:10.5009/ gnl14391

31. Sporea I, Bota S, Sirli R, et al. 1072 Acoustic Radiation Force Impulse (ARFI) Elastography for the Assessment of Liver Fibrosis in Patients with Chronic Hepatitis B and C - An International Study. J Hepatol 2012;56 Suppl 2:S421-S422. doi:10.1016/s0168-8278(12)61084-8

32. Tai DI, Tsay PK, Jeng WJ, et al. Differences in liver fibrosis between patients with chronic hepatitis B and C. J Ultrasound Med 2015;34:813821. doi:10.7863/ultra.34.5.813
33. Nierhoff J, Chávez Ortiz AA, Herrmann E, Zeuzem S, Friedrich-Rust $M$. The efficiency of acoustic radiation force impulse imaging for the staging of liver fibrosis: a meta-analysis. Eur Radiol 2013;23:3040-3053. doi:10.1007/s00330-013-2927-6

34. Bedossa P, Poynard T. An algorithm for the grading of activity in chronic hepatitis C. Hepatology 1996;24:289-293. doi:10.1002/hep.510240201

35. Ishak K, Baptista A, Bianchi L, et al. Histological grading and staging of chronic hepatitis. J Hepatol 1995;22:696-699. doi:10.1016/01688278(95)80226-6

36. Bamber J, Cosgrove D, Dietrich CF, et al. EFSUMB guidelines and recommendations on the clinical use of ultrasound elastography. Part 1: Basic principles and technology. Ultraschall Med 2013;34:169-184 doi:10.1055/s-0033-1335205

37. Ferraioli G, De Silvestri A, Lissandrin R, et al. Evaluation of InterSystem Variability in Liver Stiffness Measurements. Ultraschall Med 2018;40:64-75. doi:10.1055/s-0043-124184

38. Myers R, Pomier-Layrargues G, Kirsch R, et al. Discordance in fibrosis staging between liver biopsy and transient elastography using the FibroScan XL probe. J Hepatol 2012;56:564-570. doi:10.1016/j. jhep.2011.10.007

39. Myers RP, Crotty P, Pomier-Layrargues G, Ma M, Urbanski SJ, Elkashab M. Prevalence, risk factors and causes of discordance in fibrosis staging by transient elastography and liver biopsy. Liver Int 2010;30:1471-1480. doi:10.1111/j.1478-3231.2010.02331.x

40. Herrero JI, Iñarrairaegui M, D’Avola D, Sangro B, Prieto J, Quiroga J. Comparison of the M and XL FibroScan(s) probes to estimate liver stiffness by transient elastography. Gastroenterol Hepatol 2014;37:233239. doi:10.1016/j.gastrohep.2013.10.009

41. Berger A, Shili S, Zuberbuhler F, et al. Liver Stiffness Measurement with FibroScan: Use the Right Probe in the Right Conditions! Clin Transl Gastroenterol 2019;10:e00023. doi:10.14309/ctg.0000000000000023

42. Piyachaturawat P, Siramolpiwat S, Sonsiri K, Tangkijvanich P, Treeprasertsuk S. Changes in transient elastography in early cirrhotic patients after receiving nonselective B-blocker for primary variceal bleeding prophylaxis: Three-month follow up. JGH Open 2018;2:172177. doi:10.1002/jgh3.12063

43. Conti F, Vukotic R, Foschi FG, et al. Transient elastography in healthy subjects and factors influencing liver stiffness in non-alcoholic fatty liver disease: An Italian community-based population study. Dig Liver Dis 2016;48:1357-1363. doi:10.1016/j.dld.2016.07.020

44. Shan R, Yin H, Yang W, et al. Influencing factors of transient elastography in detecting liver stiffness. Exp Ther Med 2016;12:23022306. doi:10.3892/etm.2016.3617

45. Nishikawa T, Hashimoto S, Kawabe N, et al. Factors correlating with acoustic radiation force impulse elastography in chronic hepatitis C. World J Gastroenterol 2014;20:1289-1297. doi:10.3748/wjg.v20.i5.1289

46. Harris N, Nadebaum D, Christie M, et al. Acoustic radiation force impulse accuracy and the impact of hepatic steatosis on liver fibrosis staging. J Med Imaging Radiat Oncol 2016;60:587-592. doi:10.1111/1754-9485.12482

47. Rizzo L, Calvaruso V, Cacopardo B, et al. Comparison of transient elastography and acoustic radiation force impulse for non-invasive staging of liver fibrosis in patients with chronic hepatitis C. Am J Gastroenterol 2011;106:2112-2120. doi:10.1038/ajg.2011.341

48. Ziol M, Handra-Luca A, Kettaneh A, et al. Noninvasive assessment of liver fibrosis by measurement of stiffness in patients with chronic hepatitis C. Hepatology 2004;41:48-54. doi:10.1002/hep.20506

49. Castéra L, Vergniol J, Foucher J, et al. Prospective comparison of transient elastography, Fibrotest, APRI, and liver biopsy for the 
assessment of fibrosis in chronic hepatitis C. Gastroenterology 2005;128:343-350. doi:10.1053/j.gastro.2004.11.018

50. Ragazzo T, Paranagua-Vezozzo D, Lima FR, et al. Accuracy of transient elastography-FibroScan ${ }^{\circ}$, acoustic radiation force impulse (ARFI) imaging, the enhanced liver fibrosis (ELF) test, APRI, and the FIB-4 index compared with liver biopsy in patients with chronic hepatitis C. Clinics (Sao Paulo) 2017;72:516-525. doi:10.6061/ clinics/2017(09)01

51. Friedrich-Rust M, Nierhoff J, Lupsor M, et al. Performance of Acoustic Radiation Force Impulse imaging for the staging of liver fibrosis: a pooled meta-analysis. J Viral Hepat 2012;19:e212-e219. doi:10.1111/ j.1365-2893.2011.01537.x
52. Lee MS, Bae J, Joo SK, et al. Prospective comparison among transient elastography, supersonic shear imaging, and ARFI imaging for predicting fibrosis in nonalcoholic fatty liver disease. PLoS One 2017;12:e0188321. doi:10.1371/journal.pone.0188321

53. Sporea I, Badea R, Șirli R, et al. How efficient is acoustic radiation force impulse elastography for the evaluation of liver stiffness? Hepat Mon 2011;11:532-538.

54. Ryu H, Ahn SJ, Yoon JH, Lee JM. Inter-platform reproducibility of liver stiffness measured with two different point shear wave elastography techniques and 2-dimensional shear wave elastography using the comb-push technique. Ultrasonography 2019;38:345-354. doi:10.14366/ usg. 19001 